\title{
Fatty acid-binding protein 1 is preferentially lost in microsatellite instable colorectal carcinomas and is immune modulated via the interferon $y$ pathway
}

Stephanie M Wood ${ }^{1}$, Anthony J Gill ${ }^{2}$, Alexander S Brodsky ${ }^{1}$, Shaolei Lu ${ }^{1}$, Kenneth Friedman ${ }^{1}$, Galina Karashchuk ${ }^{1}$, Kara Lombardo ${ }^{1}$, Dongfang Yang ${ }^{1}$ and Murray B Resnick ${ }^{1}$

${ }^{1}$ Department of Pathology and Laboratory Medicine, Rhode Island Hospital and Alpert Medical School of
Brown University, Providence, RI, USA and ${ }^{2}$ Cancer Diagnosis and Pathology Research Group, Kolling
Institute of Medical Research, Royal North Shore Hospital and University of Sydney, Sydney, NSW, Australia

Fatty acid-binding protein 1 (FABP1) is an intracellular protein responsible for the transportation of long chain fatty acids. Aside from its functions in lipid metabolism and cellular differentiation, FABP1 also plays a role in inflammation through its interaction with peroxisome proliferator-activated receptors (PPARs). Previously, we compared expression of colonic epithelium genes in a subset of microsatellite instable (MSI) colorectal carcinomas (medullary carcinomas) to normal colonic mucosa and found that FABP1 expression was markedly decreased in the tumors. Further analysis of RNA expression in the colorectal subtypes and The Cancer Genome Atlas data set found that FABP1 expression is decreased in the CMS1 subset of colorectal carcinomas, which is characterized by microsatellite instability. As MSI colorectal carcinomas are known for their robust immune response, we then aimed to link FABP1 to the immune microenvironment of MSI carcinomas. To confirm the gene expression results, we performed immunohistochemical analysis of a cohort of colorectal carcinomas. FABP1 was preferentially lost in MSI carcinomas (123/133, 93\%) compared with microsatellite stable carcinomas $(240 / 562,43 \%, P<0.0001)$. In addition, higher numbers of tumor-infiltrating lymphocytes were present in tumors with loss of FABP1 $(P<0.0001)$. Decreased expression of the fatty acid storage and glucose regulator, PPAR $\gamma$, was associated with the loss of FABP1 $(P<0.0001)$. Colorectal cancer cell lines treated with interferon $\gamma$ exhibited decreased expression of FABP1. FABP1 expression was partially recovered with the treatment of the cell lines with rosiglitazone, a PPARy agonist. This study demonstrated that the loss of FABP1 expression is associated with MSI carcinomas and that interferon $\gamma$ stimulation plays a role in this process via its interaction with PPAR $y$. Modern Pathology (2017) 30, 123-133; doi:10.1038/modpathol.2016.170; published online 30 September 2016

Colorectal carcinoma is a heterogeneous disease with multiple molecular pathways leading to tumorigenesis, many of which have prognostic and predictive significance. In routine practice, colorectal carcinomas are assessed for microsatellite instability, which is characterized by the deficiency of mismatch repair proteins and a hypermutator phenotype. Microsatellite instable (MSI) carcinomas

Correspondence: Dr MB Resnick, MD, PhD, Department of Pathology and Laboratory Medicine, Rhode Island Hospital and Alpert Medical School of Brown University, 593 Eddy Street, Providence, RI 02903, USA.

E-mail: mresnick@lifespan.org

Received 27 May 2016; revised 22 August 2016; accepted 23

August 2016; published online 30 September 2016 typically have a better prognosis than microsatellite stable (MSS) carcinomas and are characterized by a robust immune microenvironment with the increased tumor-infiltrating lymphocytes and increased inflammatory cytokines (including interferon $\gamma$ ). ${ }^{1-3}$ New classification systems in which MSI status plays a prominent role have been proposed to classify colorectal carcinomas based on the gene expression analysis in order to better predict prognosis and select patients for adjuvant therapy. ${ }^{3-5}$

We initially aimed to examine genes differentially expressed in a subset of MSI tumors (medullary carcinomas) and found expression of the fatty acid-binding protein 1 gene $(F A B P 1)$ to be significantly decreased, as compared with adjacent normal mucosa. ${ }^{6}$ FABP1, also called liver FABP is an 
intracellular protein important in the transport of long chain fatty acids. FABP1 is a normal component of hepatocytes, enterocytes of the colon and small bowel, and is present to a lesser degree in tubular cells of the kidney and alveolar cells in the lung. ${ }^{7}$ It has been shown to be a marker of differentiation of colon enterocytes. ${ }^{8}$ The FABP family is involved in the lipid metabolism, but also plays a role in the regulation of inflammation and cellular metabolism via interaction with peroxisome proliferatoractivated receptors (PPARs). ${ }^{9-14}$ FABP1 has been shown to directly interact with PPAR $\gamma$ in the nucleus to activate its downstream transcriptional targets, many of which are involved in the cellular differentiation, apoptosis, and anti-inflammatory response. ${ }^{15,16}$ In addition to activating PPAR $\gamma, \mathrm{FABP} 1$ is also a downstream transcriptional target of PPAR $\gamma$, suggesting a delicate feedback loop involving inflammation and cellular proliferation. ${ }^{7,13,15}$

FABP1 and PPAR $\gamma$ have been studied in various carcinomas including colorectal carcinoma. Loss of PPAR $\gamma$ in colorectal carcinoma has been described, with conflicting prognostic significance. ${ }^{17-19}$ FABP1 has been shown to be upregulated in some carcinomas, such as carcinomas of the prostate and pancreas, although being lost in others. ${ }^{20,21}$ Loss of FABP1 has been described in colorectal carcinomas, though with conflicting prognostic data. ${ }^{5,20,22,23}$ However, FABP1 expression has not been evaluated with respect to MSI status. In this study, we further characterize FABP1 expression in MSS vs MSI tumors and associate its expression with clinicopathologic features and PPAR $\gamma$ expression. We also performed in vitro studies to better clarify the relationship between FABP1 expression, immune pathways, and PPAR $\gamma$.

\section{Materials and methods}

\section{Patients and Samples}

Archive formalin-fixed paraffin-embedded samples of colorectal carcinoma were collected by the Royal North Shore Hospital, St Leonards Australia. Sample selection has been described previously and is summarized here. ${ }^{24}$ Briefly, the computerized colorectal specimen database maintained by the Department of Anatomical Pathology at the Royal North Shore Hospital, Sydney, was searched to identify all cases of colorectal carcinomas that underwent nonendoluminal resection from 2007 to 2009. Cases other than colorectal adenocarcinoma and its variants were excluded, as were those carcinomas arising in the appendix. This database was current with all-cause survival data and all cases were staged according to the American Joint Committee on Cancer, 7th edition. A total of 722 colorectal carcinomas were analyzed: 576 MSS carcinomas and 133 MSI carcinomas (including 25 medullary carcinomas).
All-cause survival data in the database were derived from the examination of hospital medical and pathology records, medical records from the surgeons' private rooms, and publically available death notices. Survival was calculated from the date of surgical resection to the last known date of contact or notification of death.

Tissue microarrays were constructed of two 1-mm cores from all colorectal carcinomas with available paraffin-embedded tissue from 2007 to 2009. Only adenocarcinomas were included in the study; neuroendocrine carcinomas and other malignancies were excluded.

\section{Gene Expression Analysis}

The medullary vs normal tumor data was previously published $^{6}$ and the data are available in Gene Expression Omnibus (GSE76855). We evaluated the signals for the FABP family. Normalized HiSeq RNAseq data for colorectal tumors from The Cancer Genome Atlas were downloaded from Firebrowse in September $2015 .{ }^{6}$ Clinical data were downloaded from the The Cancer Genome Atlas data portal (https://tcga.data.nci.gov/tcga/dataAccessMatrix.htm, download in September 2015). Molecular subtypes (MSI-H, MSI-L, and MSS) were acquired in The Cancer Genome Atlas clinical data. The authors' annotation for the proposed molecular subtypes by Guinney et $a l^{4}$ was used. Functional analyses were performed using Gene Set Enrichment Analysis. ${ }^{25}$ Fold change, $t$-tests, and multiple hypothesis tests were calculated in $\mathrm{R}$ version 3.1.1.

\section{Immunohistochemistry}

Table 1 contains a summary of the immunohistochemical stains used. Briefly, stains for each antigen were performed on $5 \mu \mathrm{m}$ paraffin sections of each tissue microarray masterblock. The panel included antibodies against CD3 (rabbit polyclonal, ready to use, Dako, Carpinteria, CA), FABP1 (mouse monoclonal, 1:1000, Abcam, Cambridge, MA, USA), and PPAR $\gamma$ (rabbit polyclonal, 1:50, Abcam). The stains were run on the Dako autostainer using the Envision Plus kit (Dako). Slides were counterstained with hematoxylin, dehydrated, and coverslipped. Positive controls included normal colon (PPAR), normal tonsil (CD3), and normal liver (FABP).

\section{Immunohistochemical Assessment and Scoring}

Immunohistochemical stains for FABP1 and PPAR $\gamma$ were evaluated in tumor epithelium. CD3 was used to evaluate numbers of intratumoral infiltrating lymphocytes. For FABP1 and PPAR $\gamma$, epithelial staining was considered positive in cases with $>10 \%$ moderate-to-intense staining in tumor epithelium. Staining was scored as negative for tumors with $<10 \%$ moderate-to-intense staining. Tumors 
Table 1 Immunohistochemical markers, clones, and protocols

\begin{tabular}{|c|c|c|c|c|c|}
\hline Antibody & Vendor & Host & Antigen retrieval & Dilution & Detection method \\
\hline $\mathrm{PPAR} \gamma$ & $\begin{array}{l}\text { Abcam } \\
\text { Cambridge, MA }\end{array}$ & Rabbit polyclonal & $\begin{array}{l}\text { EDTA pH } 9 \\
\text { Microwave } \\
120^{\circ} \mathrm{C}, 10 \mathrm{~min}\end{array}$ & $1: 50$ & Dako Envision+Kit \\
\hline CD3 & $\begin{array}{l}\text { Dako } \\
\text { Carpinteria, CA }\end{array}$ & Rabbit polyclonal & $\begin{array}{l}\text { EDTA pH } 9 \\
\text { Dako PTLink } \\
97^{\circ} \mathrm{C}, 20 \text { min }\end{array}$ & RTU & Dako Envision FLEX Autostainer \\
\hline FABP1 & $\begin{array}{l}\text { Abcam, } \\
\text { Cambridge, MA }\end{array}$ & $\begin{array}{l}\text { Mouse monoclonal } \\
\text { Clone 2G4 }\end{array}$ & $\begin{array}{l}\text { EDTA pH } 9 \\
\text { Dako PTLink } \\
97^{\circ} \mathrm{C}, 20 \text { min }\end{array}$ & $1: 1000$ & Dako Envision FLEX Autostainer \\
\hline
\end{tabular}

Abbreviations: FABP1, fatty acid-binding protein 1; PPAR $\gamma$, proliferator-activated receptor $\gamma$; RTU, ready to use.

with less than moderate staining (including absence of staining) were included in the negative-staining group. These cutoffs were determined and scored by a single observer (SW) to minimize the interobserver variability. Intraepithelial lymphocytes positive for CD3 were manually counted and the counts per core were averaged for each tumor.

\section{Cell Culture}

The human epithelial colorectal adenocarcinoma cell lines HT-29 (HTB-38) and Colo 205 (CCL-222) were obtained from American Type Culture Collection (ATCC, Manassas, VA, USA). Cells were maintained in RPMI-1640 medium (Life Technologies) containing $2 \mathrm{~g} / \mathrm{l}$ D-glucose and $2 \mathrm{mM}$ glutamine, and supplemented with $10 \%(\mathrm{v} / \mathrm{v})$ fetal bovine serum, $100 \mathrm{U} / \mathrm{ml}$ penicillin, and $100 \mu \mathrm{g} / \mathrm{ml}$ streptomycin. All cells were grown at $37{ }^{\circ} \mathrm{C}$ and $5 \%$ CO2. Cells were treated for $24 \mathrm{~h}$ with $0.02 \%$ DMSO and 1\% BSA, interferon gamma (IFN $\gamma ; 10 \mathrm{ng} / \mathrm{ml})$, rosiglitazone $(10 \mu \mathrm{M})$, or the combination of both. IFN $\gamma$ and rosiglitazone were purchased from Sigma-Aldrich, Saint Louis, MO, USA.

\section{Quantitative Real-Time PCR}

Total RNA was isolated from cells using RNeasy mini-kit (Qiagen, Valencia, CA, USA) following the manufacturer's instructions. The total RNA concentration and purity were determined using Nano drop spectrophotometer at $260 \mathrm{~nm}$ absorbance. Reverse transcription was conducted with Maxima First Strand cDNA Synthesis Kit for RT-qPCR (ThermoFisher Scientific, Houston, TX, USA) on $1 \mu \mathrm{g}$ of total RNA in a final reaction volume of $20 \mu \mathrm{l}$ according to the manufacturer's instructions. Quantitative realtime PCR (qRT-PCR) was conducted in a final volume of $25 \mu \mathrm{l}$ using SYBR Green/ROX qPCR Master Mix $(2 \times$; ThermoFisher Scientific) for absolute gene quantification. All reactions were performed in duplicates. Values were normalized to actin and relative levels of mRNA expression were calculated by the $2^{-\Delta \Delta C T}$ method. The primer sequences used in this study are listed in Supplementary Table 1. Data are represented as mean or mean of $\log 10$ values \pm s.d. of three biological replicates.

\section{Statistical Analysis}

The $\chi^{2}$-analysis was used to assess the immunohistochemical staining scores. One-way variance analysis and $t$-test were used for comparing lymphocyte counts among tumor types and between other characteristics. Cox proportional hazard analysis was used to evaluate the risk ratios in univariate and multivariate analysis. All tests were two-sided with 0.05 as the threshold $P$-value to be considered statistically significant. All analyses were performed using the SAS software, JMP Pro version 11.1 (SAS, Cary, NC, USA).

\section{Results}

\section{Gene Expression Results}

Of the 32670 genes represented on the microarrays, 7429 were differentially expressed $(P<0.05)$ between medullary carcinoma and adjacent histologically normal colonic mucosa in all six cases. FABP1 was decreased in medullary carcinoma vs normal colonic epithelium by 55 -fold $(P<0.001$, FDR $=0.7)$. PPAR was decreased by twofold $(P=0.03, \mathrm{FDR}=0.2)$.

Among all tumors, FABP1 expression was associated with $P P A R \gamma$ expression in the Hiseq The Cancer Genome Atlas dataset (Rho 0.47, $P<0.0001$ ). FABP1 and PPARy expression were also associated in MSI and MSS subgroups (Rho 0.47 and 0.48, respectively, $P<0.0001$ for both subgroups). PPAR $\alpha$ expression was not statistically associated with $F A B P 1$ expression in colon carcinomas in The Cancer Genome Atlas dataset $(P=0.2$ for all tumors, $P=0.6$ for MSS tumors, and $P=0.1$ for MSI tumors).

\section{Consensus Molecular Subtype Comparison}

Four consensus molecular subtypes of colorectal carcinomas were recently proposed. ${ }^{4}$ We assessed the expression of FABP1 and PPAR in these subtypes in The Cancer Genome Atlas RNA-seq data. 

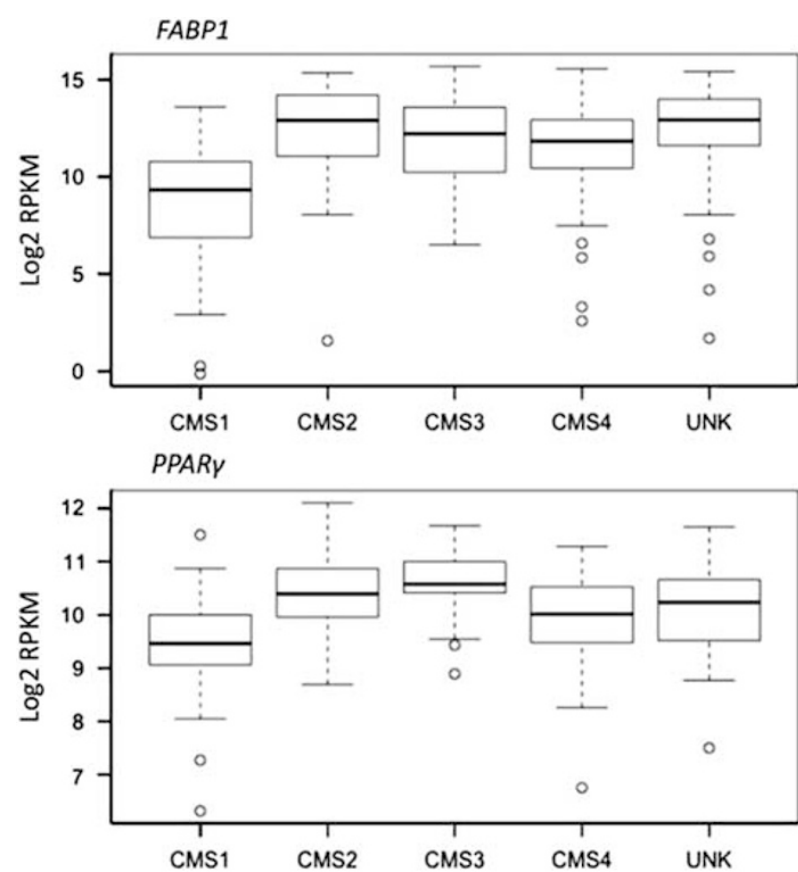

Figure $1 F A B P 1$ and PPAR expression in The Cancer Genome Atlas HiSeq colorectal carcinoma data was distributed across the pre-assigned classification of colorectal carcinoma subtypes according to Guinney et al. ${ }^{4} F A B P 1$ and PPAR show decreased expression in the microsatellite instability immune subgroup (CMS1) when compared with the other categories. FABP1, fatty acid-binding protein 1; PPAR $\gamma$, proliferator-activated receptor $\gamma$; UNK, unknown subtype.

Two-hundred thirty three colorectal carcinomas with known CMS group classification were compared for FABP1 and PPAR expression. The CMS1 carcinomas had decreased FABP1 and PPAR expression when compared with the other CMS groups (CMS2, CMS3, and CMS4) and carcinomas of unknown CMS group (Figure 1). CMS1 is enriched for MSI cases and high immune cell infiltration. These observations show that expression of FABP1 and PPAR $\gamma$ is decreased in tumors with MSI.

\section{Clinicopathologic Features}

The clinicopathologic features of patients and their tumors included in the tissue microarray are summarized in Table 2. Tumor site and grade were unavailable for two and three patients, respectively. Tumor size was unavailable for two patients. The average age at time of surgery was 72 years (77 years for patients with MSI tumors and 71 year for MSS tumors). A majority of the patients with MSI tumors were female $(72 \%$ female) compared with MSS tumors $(48 \%$ female, $P<0.0001)$, and MSI tumors were more often located in the right colon $69 \%$ right sided) when compared with MSS tumors (31\% right sided, $P<0.0001)$. MSI tumors presented at a lower stage $(70 \%$ in stage I-II) than MSS tumors ( $47 \%$ stage I-II, $P<0.0001$ ), though on average MSI tumors were larger in size (5.1 vs $4.2 \mathrm{~cm}, P<0.0001)$. MSI tumors were less likely to have lymphovascular invasion (present in $37 \%$ of MSI vs $49 \%$ of MSS tumors, $P=0.01)$. Lymph node metastasis was more common in MSS tumors (49 vs $29 \%, P<0.0001$ ).

\section{FABP1 Protein Expression and Correlation with Other Clinicopathologic Features}

On the basis of the gene expression results that found $F A B P 1$ to be decreased in medullary carcinomas (a subset of MSI carcinomas), we decided to test a panel of colorectal carcinomas for protein FABP1 expression via immunohistochemistry. FABP1 stained in a cytoplasmic and nuclear pattern in tumor and normal colorectal epithelial cells (Figure 2). Staining in normal epithelium was confined to the surface epithelial cells, similar to previously reported staining patterns. ${ }^{11}$ There was loss of expression of FABP1 in $52 \%$ of all tumors tested (Figure 3 ). The majority of MSI tumors showed loss of FABP1 (93\%), whereas only $43 \%$ of MSS tumors had loss of FABP1 staining $(P<0.0001$, Figure 3$)$. FABP1 was lost in $96 \%$ of medullary carcinomas, compared with $59 \%$ of nonmedullary carcinomas (including non-medullary MSI carcinomas and MSS carcinomas, $P=0.0003$ ).

Loss of FABP1 expression in all colorectal carcinomas associated significantly with older age $(P<0.0001)$, right-sided location of tumor $(P<0.0001)$, and higher grade $(P<0.0001$, Table 3$)$. Within the MSI tumors, loss of FABP1 expression associated significantly with right-sided location $(P=0.001)$. Within the MSS tumors, loss of FAPB1 expression associated significantly with older age $(P=0.003$, Table 3$)$.

\section{$\operatorname{PPAR} \gamma$ Expression and Correlation with Other Clinicopathologic Features}

We hypothesized that FABP1 loss may be linked to MSI tumors via inflammatory pathways that include the PPAR $\gamma$ protein. ${ }^{9-13}$ Therefore, we tested for PPAR $\gamma$ expression in our colorectal carcinoma cohort via immunohistochemistry. PPAR $\gamma$ stained in a nuclear pattern in tumor and normal colorectal epithelial cells. Loss of PPAR $\gamma$ was seen in $11 \%$ of all tumors and was more common in MSI over MSS tumors (22 and $8.8 \%$ respectively, $P<0.0001$; Figure 3 ).

Loss of PPAR $\gamma$ in all colorectal carcinomas was associated with right-sided location $(P=0.03)$ and lower stage $(P=0.04$; Table 3$)$. Within the MSI and MSS tumors, PPAR $\gamma$ did not significantly associate with clinicopathologic features.

\section{CD3 Tumor Infiltrating Lymphocytes and Correlation with Other Clinicopathologic Features}

Because our hypothesis linked FABP1 loss to MSI carcinomas via immune pathways, we decided to evaluate the number of tumor-infiltrating lymphocytes in our cohort. CD3 immunohistochemistry was 
Table 2 Clinicopathologic features of study population

\begin{tabular}{|c|c|c|c|c|}
\hline Clinicopathologic features & All tumors (695) & $M S I(\mathrm{n}=133)$ & $M S S(\mathrm{n}=562)$ & P-value \\
\hline \multicolumn{5}{|l|}{ Age at surgery } \\
\hline Mean & 72 & 77 & 71 & \multirow[t]{2}{*}{$<0.0001$} \\
\hline Range & $33-99$ & $41-97$ & $33-99$ & \\
\hline \multicolumn{5}{|l|}{ Sex } \\
\hline Male & $330(47 \%)$ & $37(28 \%)$ & $293(52 \%)$ & \multirow[t]{2}{*}{$<0.0001$} \\
\hline Female & $365(53 \%)$ & $96(72 \%)$ & $269(48 \%)$ & \\
\hline \multicolumn{5}{|l|}{ Location $^{\mathrm{a}}$} \\
\hline Right & $263(38 \%)$ & $91(69 \%)$ & $172(31 \%)$ & \multirow{3}{*}{$<0.0001$} \\
\hline Transverse & $66(10 \%)$ & $30(23 \%)$ & $36(6 \%)$ & \\
\hline Left & $364(52 \%)$ & $11(8 \%)$ & $353(63 \%)$ & \\
\hline \multicolumn{5}{|l|}{ Gradeb } \\
\hline Low & $549(79 \%)$ & $78(59 \%)$ & $471(84 \%)$ & \multirow[t]{2}{*}{$<0.0001$} \\
\hline High & $143(21 \%)$ & $54(41 \%)$ & $89(16 \%)$ & \\
\hline \multicolumn{5}{|l|}{ Stage } \\
\hline I & $112(16 \%)$ & $16(12 \%)$ & $96(17 \%)$ & \multirow[t]{4}{*}{$<0.0001$} \\
\hline II & $245(35 \%)$ & $77(58 \%)$ & $168(30 \%)$ & \\
\hline III & $312(45 \%)$ & $39(29 \%)$ & $273(49 \%)$ & \\
\hline IV & $26(4 \%)$ & $1(1 \%)$ & $25(4 \%)$ & \\
\hline Tumor size & $4.4 \mathrm{~cm}$ & 5.1 & 4.2 & $<0.0001$ \\
\hline \multicolumn{5}{|l|}{ Lymphovascular invasion } \\
\hline Present & $325(47 \%)$ & $49(37 \%)$ & $276(49 \%)$ & \multirow[t]{2}{*}{0.01} \\
\hline Absent & $370(53 \%)$ & $84(63 \%)$ & $286(51 \%)$ & \\
\hline \multicolumn{5}{|l|}{ Lymph node metastasis } \\
\hline Present & $313(45 \%)$ & $39(29 \%)$ & $274(49 \%)$ & \multirow[t]{2}{*}{$<0.0001$} \\
\hline Absent & $382(55 \%)$ & $94(71 \%)$ & $288(51 \%)$ & \\
\hline
\end{tabular}

Abbreviations: MSI, microsatellite instable; MSS, microsatellite stable.

${ }^{\text {a }}$ Site missing for two cases (one MSI case and one MSS case).

${ }^{\mathrm{b}}$ Grade missing for three cases (one MSI case and two MSS cases).

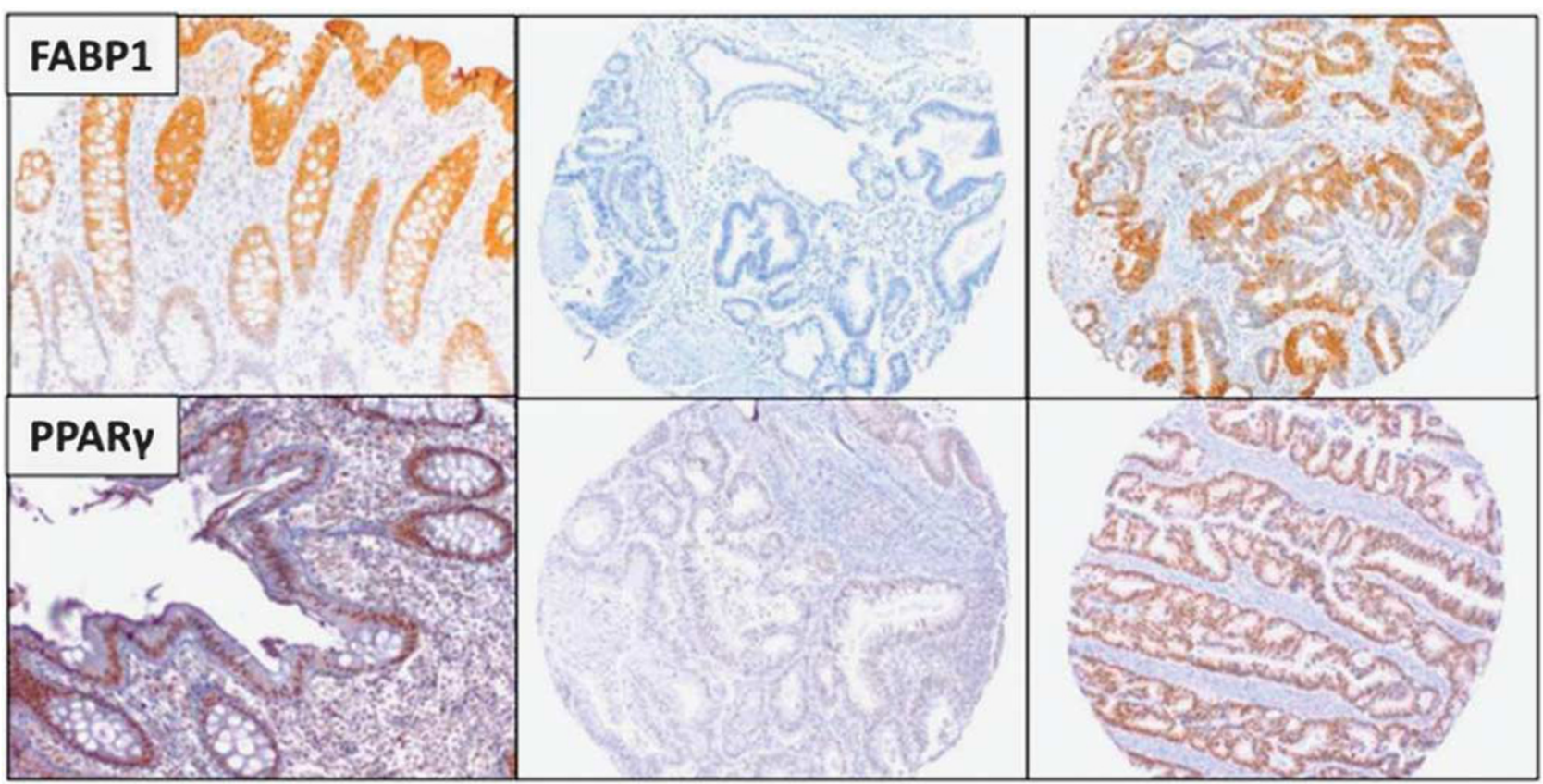

Figure 2 Representative immunohistochemistry staining of normal colonic epithelium, positive staining colorectal carcinomas, and negative staining colorectal carcinomas for FABP1 and PPAR $\gamma$. Left column, normal colonic epithelium; middle column, negative staining pattern; right column, positive staining pattern; FABP1, fatty acid-binding protein 1; PPAR $\gamma$, proliferator-activated receptor $\gamma$. 
utilized to count intratumoral lymphocytes. As expected, MSI tumors demonstrated higher average tumor-infiltrating lymphocytes than MSS tumors (151 and 35 respectively, $P<0.0001$; Figure 5).

Among all colorectal carcinomas, higher numbers of tumor-infiltrating lymphocytes associated significantly with right-sided location $(P<0.0001)$, absence of lymphovascular invasion $(P=0.04)$, lower stage $(P<0.0001)$, and high grade $(P<0.0001$; Table 4$)$.

Within MSI tumors, higher-tumor-infiltrating lymphocytes associated significantly with high grade $(P=0.02)$. In MSS tumors, higher numbers of tumorinfiltrating lymphocytes associated significantly with

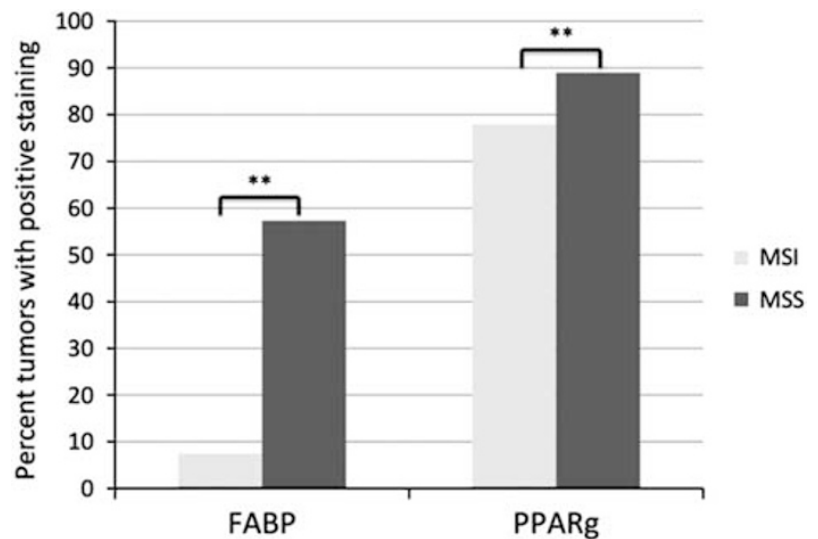

Figure 3 FABP1 expression is decreased in MSI colorectal carcinomas over MSS colorectal carcinomas. Overall expression and paired data for immunohistochemistry expression results in MSI compared with MSS colorectal carcinomas. **Statistically highly significant as $P<0.001$. FABP1, fatty acid-binding protein 1; MSI, microsatellite instable; MSS, microsatellite stable. the absence of lymphovascular invasion $(P=0.0005)$, and lower stage $(P<0.0001)$.

\section{Correlation of FABP1 with PPAR $\gamma$ and Tumor-Infiltrating Lymphocytes}

We hypothesized that loss of FABP1 was due to the increased inflammation associated with MSI tumors. We therefore expected FABP1 to be decreased in tumors with increased tumor-infiltrating lymphocytes. As a part of the proposed pathway, we expected decreased FABP1 to be associated with decreased PPAR $\gamma$. Our data showed that in all colorectal carcinomas, loss of expression of FABP1 significantly associated with loss of PPAR $\gamma(P<0.0001)$ and increased tumor-infiltrating lymphocytes $(P<0.0001$; Figure 4 and Figure 5).

Within MSI colorectal carcinomas, loss of FABP1 expression significantly associated with the loss of PPAR $\gamma$ $(P=0.03)$. Though not statistically significant, MSI tumors with loss of FABP1 showed increased tumor-infiltrating lymphocytes (74 in FABP-positive MSI tumors vs 157 in FABP-negative MSI tumors, $P=0.2$ ). Likely, the lack of statistical significance is due to the low numbers of MSI tumors that are also FABP positive. Within MSS colorectal carcinomas, loss of FABP1 expression significantly associated with loss of $\operatorname{PPAR} \gamma(P=0.02)$ expression.

\section{IFN $\gamma$ Downregulates and Stimulation of PPAR $\gamma$ Upregulates FABP1 Expression}

Increased tumor-infiltrating lymphocytes in MSI tumors is associated with the IFN $\gamma$ pathway. ${ }^{2}$ In addition, IFN $\gamma$ response was significantly associated

Table 3 Clinicopathologic features in relation to staining characteristics of colorectal carcinomas in all cases, MSI cases, and MSS cases

\begin{tabular}{|c|c|c|c|c|c|c|c|c|c|c|c|c|c|c|c|}
\hline & \multicolumn{2}{|c|}{ Age } & \multicolumn{2}{|c|}{ Gender } & \multicolumn{2}{|c|}{ Location } & \multicolumn{2}{|c|}{$\begin{array}{c}\text { Lymphovascular } \\
\text { invasion }\end{array}$} & \multicolumn{5}{|c|}{ Stage (\%) } & \multicolumn{2}{|c|}{ Grade } \\
\hline & Mean age & $\mathrm{P}$-value & Female (\%) & $\mathrm{P}$-value & Right (\%) & $\mathrm{P}$-value & Present (\%) & $\mathrm{P}$-value & 1 & 2 & 3 & 4 & P-value & High (\%) & P-value \\
\hline \multicolumn{16}{|c|}{$F A B P$} \\
\hline \multicolumn{16}{|c|}{ All cases } \\
\hline+ & 70 & \multirow[t]{2}{*}{$<0.0001$} & 50 & 0.2 & 25 & \multirow[t]{2}{*}{$<0.0001$} & 51 & \multirow[t]{2}{*}{0.06} & 15 & 31 & 50 & 4 & \multirow[t]{2}{*}{0.09} & 14 & \multirow[t]{2}{*}{$<0.0001$} \\
\hline - & 74 & & 55 & & 49 & & 44 & & 17 & 39 & 41 & 4 & & 27 & \\
\hline \multicolumn{16}{|l|}{ MSI } \\
\hline+ & 72 & \multirow[t]{2}{*}{0.2} & 89 & 0.2 & 56 & \multirow[t]{2}{*}{0.001} & 38 & \multirow[t]{2}{*}{0.6} & 0 & 67 & 33 & 0 & \multirow[t]{2}{*}{0.5} & 6 & \multirow[t]{2}{*}{0.6} \\
\hline - & 77 & & 71 & & 70 & & 30 & & 13 & 57 & 29 & 1 & & 8 & \\
\hline \multicolumn{16}{|l|}{ MSS } \\
\hline+ & 70 & \multirow[t]{2}{*}{0.003} & 49 & 0.7 & 25 & \multirow[t]{2}{*}{0.2} & 47 & \multirow[t]{2}{*}{0.3} & 16 & 30 & 50 & 4 & \multirow[t]{2}{*}{0.6} & 13 & \multirow[t]{2}{*}{0.07} \\
\hline- & 73 & & 57 & & 38 & & 52 & & 19 & 30 & 46 & 5 & & 19 & \\
\hline \multicolumn{16}{|l|}{ PPAR $\gamma$} \\
\hline All c & ses & & & & & & & & & & & & & & \\
\hline+ & 72 & \multirow[t]{2}{*}{0.1} & 53 & 0.8 & 36 & \multirow{2}{*}{0.03} & 47 & \multirow[t]{2}{*}{1.0} & 17 & 33 & 46 & 4 & \multirow[t]{2}{*}{0.04} & 20 & 0.08 \\
\hline - & 74 & & 51 & & 51 & & 47 & & 10 & 50 & 37 & 3 & & 29 & \\
\hline MSI & & & & & & & & & & & & & & & \\
\hline+ & 76 & 0.8 & 73 & 0.7 & 68 & 0.7 & 34 & 0.7 & 13 & 56 & 32 & 0 & 0.2 & 40 & 0.6 \\
\hline - & 77 & & 69 & & 72 & & 38 & & 10 & 66 & 21 & 3 & & 45 & \\
\hline MSS & & & & & & & & & & & & & & & \\
\hline+ & 71 & 0.3 & 49 & 0.3 & 30 & 0.4 & 55 & 0.4 & 18 & 29 & 49 & 5 & 0.2 & 16 & 0.6 \\
\hline - & 73 & & 41 & & 39 & & 49 & & 10 & 41 & 47 & 2 & & 19 & \\
\hline
\end{tabular}

Abbreviations: FABP1, fatty acid-binding protein 1; MSI, microsatellite instable; MSS, microsatellite stable. 


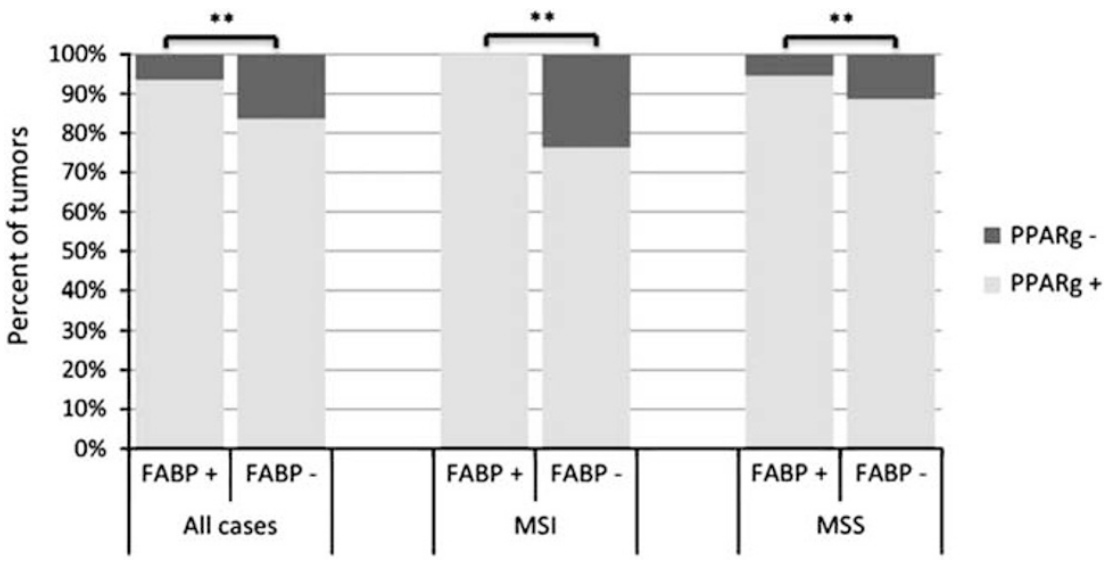

Figure 4 Average number of tumor infiltrating lymphocytes in colorectal carcinomas compared with their staining pattern in all cases, MSS cases, and MSI cases. * ${ }^{*}$ Statistically significant as $P<0.05$. MSI, microsatellite instable; MSS, microsatellite stable..

Table 4 Average count of tumor-infiltrating lymphocytes in relation to clinicopathologic features.

\begin{tabular}{|c|c|c|c|c|c|c|c|c|c|c|c|c|c|c|c|c|c|}
\hline & \multicolumn{3}{|c|}{ Gender } & \multicolumn{3}{|c|}{ Location } & \multicolumn{3}{|c|}{$L V I$} & \multicolumn{5}{|c|}{ Stage } & \multicolumn{3}{|c|}{ Grade } \\
\hline & $F$ & $M$ & $\mathrm{P}$-value & $R$ & $L$ & $\mathrm{P}$-value & Present & Absent & $\mathrm{P}$-value & 1 & 2 & 3 & 4 & P-value & Low & High & $\mathrm{P}$-value \\
\hline \multicolumn{18}{|c|}{ Tumor infiltrating lymphocytes (average) } \\
\hline All cases & 64 & 49 & 0.06 & 78 & 40 & $<0.0001$ & 49 & 65 & 0.04 & 78 & 75 & 37 & 36 & $<0.0001$ & 47 & 90 & $<0.0001$ \\
\hline MSI & 135 & 192 & 0.1 & 205 & 154 & 0.4 & 163 & 143 & 0.6 & 206 & 152 & 127 & 95 & 0.5 & 118 & 191 & 0.02 \\
\hline MSS & 39 & 21 & 0.2 & 38 & 34 & 0.5 & 28 & 42 & 0.005 & 57 & 41 & 24 & 34 & $<0.0001$ & 35 & 28 & 0.3 \\
\hline
\end{tabular}

Abbreviations: F, female; L, left; M, male; R, right.

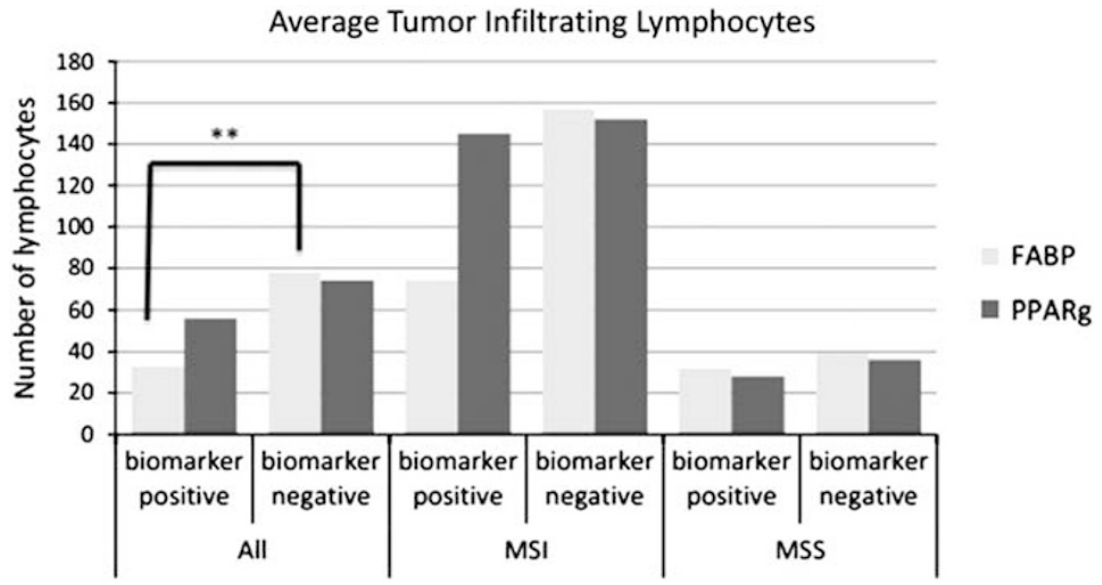

Figure 5 Comparison of staining patterns in colorectal carcinomas. Percentage of PPAR $\gamma$ negative (PPARg - ) and PPAR $\gamma$ positive (PPARg +) staining in tumors as compared with their FABP staining (in all cases, MSI cases, and MSS cases). ${ }^{*}$ Statistically highly significant as $P<0.05$. FABP1, fatty acid-binding protein 1; MSI, microsatellite instable; MSS, microsatellite stable; PPAR $\gamma$, proliferator-activated receptor $\gamma$.

with FABP1 expression in The Cancer Genome Atlas database colorectal tumor data. ${ }^{6}$ To test if IFN $\gamma$ could repress FABP1 expression, two colorectal cancer cell lines were treated with IFN $\gamma$. RT-qPCR analysis of the RNA demonstrated a significant decrease in FABP1 mRNA expression in both lines.
Rosiglitazone, a PPAR $\gamma$ agonist, reversed this inhibition and upregulated FABP1 expression. IDO and $F A S$, known targets of $\operatorname{IFN} \gamma$, served as positive controls ${ }^{26,27}$ (Figure 6). IFN $\gamma$ did not consistently induce an expression change in $P P A R \gamma$ in either cell line. 


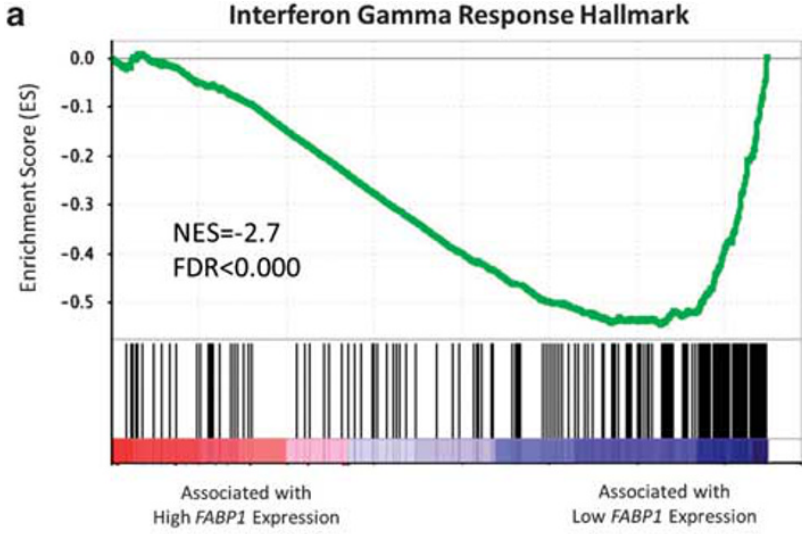

b

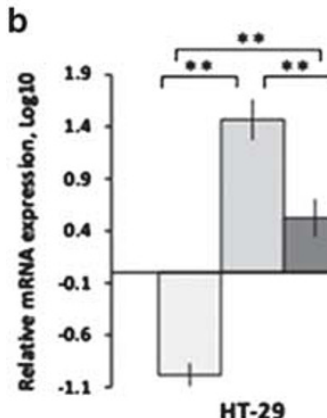

FABP1

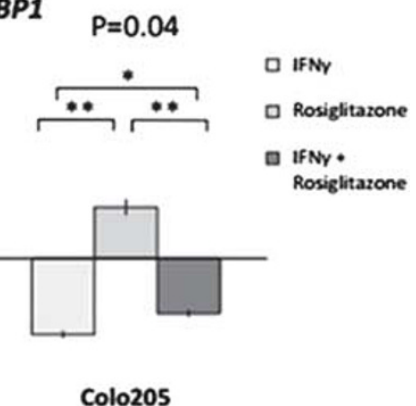

C
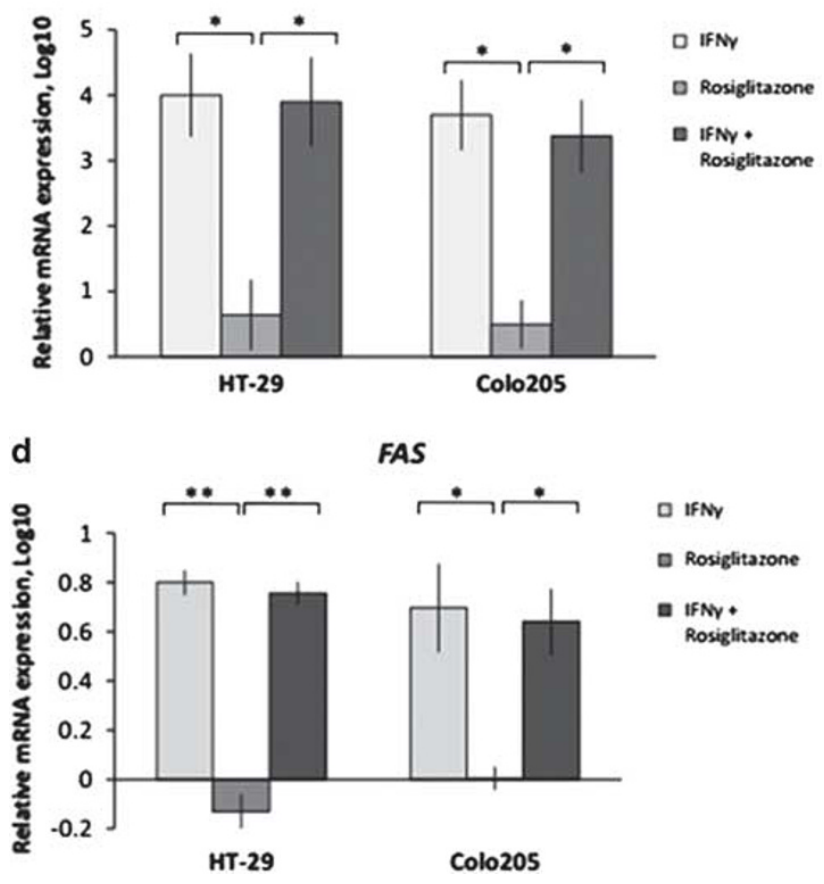

\section{Survival Data}

FABP1 and PPAR $\gamma$ expression did not correlate with survival. In a multivariate analysis, higher stage $(\mathrm{HR}=2.1,95 \%$ CI $1.6-2.9, P=<0.0001)$ and high grade $(\mathrm{HR}=1.8,95 \%$ CI $1.2-2.4, P=0.002)$ were associated with worse survival, although the
Figure 6 Interferon $\gamma$ inhibits $F A B P 1$ expression in colorectal cancer cell lines. HT-29 and Colo205 cells were treated for $24 \mathrm{~h}$ with vehicle or IFN $\gamma(10 \mathrm{ng} / \mathrm{ml})$, or rosiglitazone $(10 \mu \mathrm{M})$, or with the combination of both as indicated. (a) FABP1 mRNA expression is negatively correlated with interferon gamma response. A rank list of the Pearson correlated transcripts in the colorectal carcinoma data from The Cancer Genome Atlas was submitted to Gene Set Enrichment Analysis as a pre-rank file. The Interferon Gamma Response Hallmark was the top ranked negatively associated gene set. The FABP1 (b) and controls (c, d) mRNA expression level were determined by qRT-PCR upon treatment. The results are expressed as a fold change relative to vehicle treated cells. ${ }^{*}$ Statistically significant $P<0.05$ and ${ }^{* *}$ statistically highly significant as $P<0.001$. Error bars represent s.d. Columns represent mean of three experiments. FABP1, fatty acid-binding protein 1; IFN $\gamma$, interferon gamma; qRT-PCR, quantitative realtime PCR.

presence of microsatellite instability was associated with improved survival $(\mathrm{HR}=0.5,95 \%$ CI $0.3-0.8$, $P=0.005)$, consistent with past findings. ${ }^{28}$

\section{Discussion}

Colorectal carcinomas are a biologically diverse group of carcinomas with microsatellite instability playing an important prognostic and predictive role. MSI tumors exhibit improved survival over MSS carcinomas, which is thought to be related to the increased inflammatory response to these tumors. $^{2,28}$ Recently, gene expression studies have proposed molecular classifications of colorectal carcinomas in order to better predict prognosis and response to adjuvant therapy. However, these multigene classification systems are currently not well suited for clinical practice. ${ }^{3,4}$ Single biomarkers or small panels of biomarkers are better suited for clinical utility, if they exist.

In a previous study, we examined a subset of MSI colorectal carcinomas (medullary carcinomas) to identify genes that were differentially expressed when compared with normal colon epithelium. Many immmunoregulatory genes activated by $\mathrm{IFN} \gamma$ were upregulated in medullary colorectal carcinomas. ${ }^{6}$ Of the genes that were differentially expressed or lost in medullary carcinoma, FABP1 was shown to be decreased by 55 -fold when compared with normal colon epithelium of the same patient. In this study, we examined a larger cohort of colorectal carcinomas and showed a correlation between decreased FABP1 expression (via immunohistochemistry) and MSI tumors. This finding was supported by the finding decreased FABP1 expression in the CMS1 subgroup of colorectal carcinomas, which is characterized by microsatellite instability. As loss of FABP1 is associated with MSI colorectal carcinomas, it is not surprising that we also demonstrated decreased FABP1 to be associated with increased tumor-infiltrating lymphocytes in all cases. ${ }^{29-32} \mathrm{~A}$ similar trend was noted among MSI tumors, though this result was not statistically significant, likely due to the low numbers 
of FABP1-positive MSI cases. Interestingly, there was no correlation between increased tumor lymphocytes with FABP1 loss in the MSS cases. Although it is not clear whether the increase in tumor-infiltrating lymphocytes are directly associated with decreased FABP1 expression in the MSI population, one possible mechanism involves IFN $\gamma$ and the PPAR $\gamma$ pathway as it is well described that tumor infiltrating lymphocytes in MSI colorectal carcinomas are associated with upregulation of the IFN $\gamma$ pathway. ${ }^{2,6}$ IFN $\gamma$ expression is increased in MSI tumors when compared with MSS tumors. ${ }^{2}$ The loss of FABP1 in the MSS tumors likely does not share the same inflammatory pathway.

FABP1 has been linked to the regulation of inflammatory states via its interaction with PPARs, including PPAR $\alpha$ and PPAR $\gamma .{ }^{9-13}$ PPARs are nuclear transcription factors whose downstream effects manage lipid metabolism, inflammation, and cellular metabolism. ${ }^{13,16,32-35}$ Although FABP1 has been shown to interact with both PPAR $\alpha$ and PPAR $\gamma$ in colorectal epithelium, PPAR $\alpha$ expression was not statistically associated with FABP1 expression in colon carcinomas in The Cancer Genome Atlas data set, whereas PPAR $\gamma$ expression was associated with $F A B P 1$ expression. Also, prior studies have linked inactivation of $\mathrm{PPAR} \gamma$ with high inflammatory states. ${ }^{34}$ Therefore, we concentrated on the association of FABP1 and PPAR $\gamma$.

Because of its role in cellular metabolism including apoptosis, cellular proliferation, and angiogenesis, $\mathrm{PPAR} \gamma$ has been of interest in many carcinomas. ${ }^{17-19,22}$ Activation of PPAR $\gamma$ appears to promote apoptosis and cellular differentiation, inhibit angiogenesis, and decrease cellular proliferation. ${ }^{17} \mathrm{On}$ the basis of these properties, it would seem that increased PPAR $\gamma$ and/or PPAR $\gamma$ agonists would have anti-tumor effects. However, multiple studies have shown the opposite: increased PPAR $\gamma$ /activation of PPAR $\gamma$ actually promote carcinogenesis. Two studies involving mice with germ-line APC mutations have shown that mice treated with PPAR $\gamma$ agonists develop more colorectal carcinomas than those without PPAR $\gamma$ agonists. ${ }^{36,37}$ Similarly, mice modified with high levels of PPAR $\gamma$ in breast tissue are more likely to develop breast carcinoma. ${ }^{15}$ There have been conflicting results in retrospective studies of PPAR $\gamma$ in human colorectal carcinoma. At least one study has shown an improved survival in patients with PPAR $\gamma$-positive colorectal carcinomas in multivariate analysis, ${ }^{18}$ although others have shown no association with prognosis. ${ }^{17,19}$ PPAR $\gamma$ has been studied in other carcinomas, also with conflicting results. ${ }^{15}$ It may be that there is a balance between PPAR $\gamma$ 's anti-proliferative effects and its anti-inflammatory effects, and the tumor microenvironment may play a role in establishing PPAR $\gamma$ in a tumor suppressor or oncogenic role.

Not only does activation of PPAR $\gamma$ have downstream effects on inflammation, but it is also negatively regulated by pro-inflammatory cytokines (specifically IFN $\gamma$ and $\mathrm{TNF} \alpha$ ). PPAR $\gamma$ is bound to
RXR in the nucleus in the absence of ligand. The PPAR $\gamma-\mathrm{RXR}$ heterodimer is not transcriptionally active, and instead acts as an inhibitor when bound to DNA. In the presence of IFN $\gamma$ and TNF $\alpha$, PPAR $\gamma$ 's effects are inhibited both by decreased expression of PPAR $\gamma$ and by stabilization of the PPAR $\gamma-R X R$ heterodimer. ${ }^{33}$ FABP1 is an important part of the PPAR $\gamma$ pathway. FABP1 (with attached fatty acid ligand) has been localized to the nucleus, where it has been shown to directly interact with PPARs (including PPAR $\gamma$ ). ${ }^{14,16}$ This in turn activates PPAR $\gamma$ and results in expression of downstream transcription targets (including anti-proliferation and antiinflammatory genes). Not only can FABP1 activate $\operatorname{PPAR} \gamma$, there is also evidence that one of the downstream targets of PPAR $\gamma$ is actually FABP1 itself, forming a feedback loop. ${ }^{7,13}$ In vivo, this feedback loop is thought to decrease transcription of FABP1 when its ligands (long chain fatty acids) are scarce, and increase FABP1 during an abundance of ligand. Disruption of this balance (via a robust immune response/increased $\mathrm{IFN} \gamma$ ) may lead constitutively inactive PPAR $\gamma$ and decreased FABP1.

We proposed that the high immune response environment of MSI colorectal carcinomas may inhibit PPAR $\gamma$ via production of IFN $\gamma$ that leads to decreased transcription of FABP1. The decreased FABP1 further decreases activation of PPAR $\gamma$ through the feedback loop described above. Via immunohistochemistry, we showed that PPAR $\gamma$ expression is more likely to be decreased in colorectal carcinomas with decreased FABP1. Interestingly, PPAR $\gamma$ loss did not appear to associate with increased tumorinfiltrating lymphocytes. However, IFN $\gamma$ inhibits PPAR $\gamma$ 's transcriptional function via multiple mechanisms, including inactivation; ${ }^{33}$ therefore it is possible that the PPAR $\gamma$ present in the tumors is inactive. To further corroborate the link between IFN $\gamma$, PPAR $\gamma$, and FABP1 we performed in vitro studies using FABP1-expressing colorectal carcinoma cell lines. Cells treated with IFN $\gamma$ had decreased FABP1 expression when compared with the control. Though this suggests decreased FABP1 is due to the presence of IFN $\gamma$, this does not prove that the pathway involves PPAR $\gamma$. In fact, PPAR $\gamma$ was not decreased in the colorectal carcinoma cell lines when treated with IFN $\gamma$. However, PPAR $\gamma$ has been shown to be present, but inactive in the presence of IFN $\gamma$ in macrophages. ${ }^{33}$ To test our hypothesis that PPAR $\gamma$ plays a role in this pathway, we treated the colorectal cell line with a PPAR $\gamma$ antagonist, rosiglitazone. In the cells treated with IFN $\gamma$ and rosiglitazone, there was a modest recovery of $F A B P 1$ expression (as compared with cells treated with IFN $\gamma$ alone). These results support the hypothesis that the immune microenvironment of MSI tumors disrupts the PPAR $\gamma / \mathrm{FABP} 1$ feedback loop, leading to decreased PPAR $\gamma$ function and loss of $F A B P 1$ expression.

On the basis of FABP1's downstream effects including cellular differentiation and anti-proliferation (via its interaction with PPAR $\gamma$ ), loss of FABP1 in 
colorectal carcinomas would be expected to result in de-differentiated tumors and worse prognosis. On the other hand, FABP1 has also been shown to have antioxidant properties by reducing reactive oxygen species. ${ }^{7,38}$ Thus, decreased FABP1 could lead to increased reactive oxygen species and increased tumor cell death. Prior studies have searched for a link between FABP1 and prognosis, though results have been conflicting. Yamazaki et $a l^{23}$ showed loss of FABP1 in liver metastasis from colorectal carcinomas to be associated with decreased survival, whereas Lyall et al found that decreased FABP1 yielded better survival. However, in their same data set, their 'very poor prognosis' subgroup of colorectal carcinomas was characterized by low FABP1 expression. ${ }^{5}$ In another study, loss of FABP1 in colorectal carcinomas was associated with increased lymph node metastasis. ${ }^{22}$ FABP1 expression is particularly low in the CMS1 group in the recently proposed molecular subtypes, and CMS1 is among the better prognostic groups, compared with the clearly poor prognostic group CMS4. The link between FABP1 and microsatellite instability (through inflammatory cytokines and PPAR $\gamma$ ) may help explain the improved prognosis of MSI colorectal carcinomas via FABP1's antioxidant properties.

FABP1 expression in colorectal carcinomas did not appear to correlate with survival in our cohort; however, there were some limitations of our survival data. Many patients had only a short length of follow-up after surgery, and patient disease status (alive without disease, alive with disease, deceased) was unavailable for many. We therefore used date of last follow-up as an end point for patients with unknown data. This likely inhibited the strength of our survival data.

In conclusion, we found that FABP1 is decreased in MSI colorectal carcinomas opposed to MSS colorectal carcinomas. We provide evidence that FAPB1 loss is tied to inhibition of PPAR $\gamma$ by the immune microenvironment of MSI colorectal carcinomas. Though we were unable to relate FABP1 expression to prognostic significance in our cohort, we present a possible link between FABP1 expression and MSI colon cancers via a robust immune response and PPAR $\gamma$. This study provides additional evidence to the complex relationship between metabolic and immune pathways in carcinogenesis.

\section{Acknowledgments}

Research reported in this publication was supported by the Molecular Pathology Core of the COBRE Center for Cancer Research Development, funded by the National Institute of General Medical Sciences of the National Institutes of Health under Award Number P20GM103421. Center for Cancer Research Development, funded by the National Institute of General Medical Sciences of the National Institutes of Health under Award Number P20GM103421.

\section{Disclosure/conflict of interest}

The authors declare no conflict of interest.

\section{References}

1 Fearon ER, Vogelstein B. A genetic model for colorectal tumorigenesis. Cell 1990;61:759-767.

2 Llosa NJ, Cruise M, Tam A, et al. The vigorous immune microenvironment of microsatellite instable colon cancer is balanced by multiple counter-inhibitory checkpoints. Cancer Discov 2015;5:43-51.

3 Phipps AI, Limburg PJ, Baron JA, et al. Association between molecular subtypes of colorectal cancer and patient survival. Gastroenterology 2015;148:77-87.

4 Guinney J, Dienstmann R, Wang X, et al. The consensus molecular subtypes of colorectal cancer. Nat Med 2015;21:1350-1356.

5 Lyall MS, Dundas SR, Curran S, et al. Profiling markers of prognosis in colorectal cancer. Clin Cancer Res 2006;12:1184-1191.

6 Friedman K, Brodsky AS, Lu S, et al. Medullary carcinoma of the colon: a distinct morphology reveals a distinctive immunoregulatory microenvironment. Mod Pathol 2016;29:528-541.

7 Wang G, Bonkovsky HL, de Lemos A, et al. Recent insights into the biological functions of liver fatty acid binding protein 1. J Lipid Res 2015;56:2238-2247.

8 Trotter PJ, Storch J. Fatty acid uptake and metabolism in a human intestinal cell line (Caco-2): comparison of apical and basolateral incubation. J Lipid Res 1991;32: 293-304.

9 Boiteux G, Lascombe I, Roche E, et al. A-FABP, a candidate progression marker of human transitional cell carcinoma of the bladder, is differentially regulated by PPAR in urothelial cancer cells. Int J Cancer 2009;124:1820-1828.

10 Gajda AM, Storch J. Enterocyte fatty acid-binding proteins (FABPs): different functions of liver and intestinal FABPs in the intestine. Prostaglandins Leukot Essent Fatty Acids 2015;93:9-16.

11 Levy E, Ménard D, Delvin E, et al. Localization, function and regulation of the two intestinal fatty acid-binding protein types. Histochem Cell Biol 2009; 132:351-367.

12 Storch J, Corsico B. The emerging functions and mechanisms of mammalian fatty acid-binding proteins. Annu Rev Nutr 2008;28:73-95.

13 Varga T, Czimmerer Z, Nagy L. PPARs are a unique set of fatty acid regulated transcription factors controlling both lipid metabolism and inflammation. Biochim Biophys Acta 2011;1812:1007-1022.

14 Velkov T. Interactions between Human Liver Fatty Acid Binding Protein and Peroxisome Proliferator Activated Receptor Selective Drugs. PPAR Res 2013; 2013:938401.

15 Wang $\mathrm{T}, \mathrm{Xu}$ J, Yu X, et al. Peroxisome proliferatoractivated receptor gamma in malignant diseases. Crit Rev Oncol Hematol 2006;58:1-14.

16 Wolfrum C, Borrmann CM, Borchers T, et al. Fatty acids and hypolipidemic drugs regulate peroxisome proliferator-activated receptors alpha-and gammamediated gene expression via liver fatty acid binding protein: a signaling path to the nucleus. Proc Natl Acad Sci USA 2001;98:2323-2328. 
17 Gustafsson A, Hansson E, Kressner U, et al. EP1-4 subtype, COX and PPAR gamma receptor expression in colorectal cancer in prediction of disease-specific mortality. Int J Cancer 2007;121:232-240.

18 Ogino S, Shima K, Baba Y, et al. Colorectal cancer expression of peroxisome proliferator-activated receptor gamma (PPARG, PPARgamma) is associated with good prognosis. Gastroenterology 2009;136:1242-1250.

19 Theocharis S, Giaginis C, Parasi A, et al. Expression of peroxisome proliferator-activated receptor-gamma in colon cancer: correlation with histopathological parameters, cell cycle-related molecules, and patients' survival. Dig Dis Sci 2007;52:2305-2311.

20 Lawrie LC, Dundas SR, Curran S, et al. Liver fatty acid binding protein expression in colorectal neoplasia. Br J Cancer 2004;90:1955-1960.

21 Sharaf RN, Butte AJ, Montgomery KD, et al. Computational prediction and experimental validation associating FABP-1 and pancreatic adenocarcinoma with diabetes. BMC Gastroenterol 2011;11:5.

22 Pei $\mathrm{H}$, Zhu $\mathrm{H}$, Zeng S, et al. Proteome analysis and tissue microarray for profiling protein markers associated with lymph node metastasis in colorectal cancer. J Proteome Res 2007;6:2495-2501.

23 Yamazaki T, Kanda T, Sakai Y, et al. Liver fatty acidbinding protein is a new prognostic factor for hepatic resection of colorectal cancer metastases. J Surg Oncol 1999;72:83-87.

24 Chou A, Toon CW, Clarkson A, et al. Loss of ARID1A expression in colorectal carcinoma is strongly associated with mismatch repair deficiency. Hum Pathol 2014;45:1679-1703.

25 Subramanian A, Tamayo P, Mootha VK, et al. Gene set enrichment analysis: a knowledge-based approach for interpreting genome-wide expression profiles. Proc Natl Acad Sci USA 2005;102:15545-15550.

26 Brandacher G, Perathoner A, Ladurner R, et al. Prognostic value of indoleamine 2,3-dioxygenase expression in colorectal cancer: effect on tumor-infiltrating T cells. Clin Cancer Res 2006;12:1144-1151.

27 Schwartzberg LS, Petak I, Stewart C, et al. Modulation of the Fas signaling pathway by IFN-gamma in therapy of colon cancer: phase I trial and correlative studies of IFN-gamma, 5-fluorouracil, and leucovorin. Clin Cancer Res 2002;8:2488-2498.
28 Thibodeau SN, Bren G, Schaid D. Microsatellite instability in cancer of the proximal colon. Science 1993;260:816-819.

29 Alexander J, Watanabe T, Wu TT, et al. Histopathological identification of colon cancer with microsatellite instability. Am J Pathol 2001;158:527-535.

30 Howitt BE, Shukla SA, Sholl LM et al. Association of polymerase e-mutated and microsatellite-instable endometrial cancers with neoantigen load, number of tumor-infiltrating lymphocytes, and expression of PD-1 and PD-L1. JAMA Oncol 2015;1:1319-1323.

31 Tougeron D, Fauquembergue E, Rouquette A, et al. Tumor-infiltrating lymphocytes in colorectal cancers with microsatellite instability are correlated with the number and spectrum of frameshift mutations. Mod Pathol 2009;22:1186-1195.

32 Hughes ML, Liu B, Halls ML, et al. Fatty acid-binding proteins 1 and 2 differentially modulate the activation of peroxisome proliferator-activated receptor $\alpha$ in a ligand-selective manner. J Biol Chem 2015;290: 13895-13906.

33 Nagy ZS, Czimmerer Z, Szanto A, et al. Proinflammatory cytokines negatively regulate $\mathrm{PPAR} \gamma$ mediated gene expression in both human and murine macrophages via multiple mechanisms. Immunobiology 2013;218:1336-1344.

34 Schachtrup C, Emmler T, Bleck B, et al. Functional analysis of peroxisome-proliferator-responsive element motifs in genes of fatty acid-binding proteins. Biochem J 2004;382:239-245.

35 Tan NS, Shaw NSVinckenbosch N, et al. Selective cooperation between fatty acid binding proteins and peroxisome proliferator-activated receptors in regulating transcription. Mol Cell Biol 2002;22:5114-5127.

36 Lefebvre AM, Chen I, Desreumaux P, et al. Activation of the peroxisome proliferator-activated receptor gamma promotes the development of colon tumors in C57BL/6J-APCMin/+ mice. Nat Med 1998;4: 1053-1057.

37 Saez E, Tontonoz P, Nelson MC, et al. Activators of the nuclear receptor PPARgamma enhance colon polyp formation. Nat Med 1998;4:1058-1061.

38 Yan J, Gong Y, She YM, et al. Molecular mechanism of recombinant liver fatty acid binding protein's antioxidant activity. J Lipid Res 2009;50:2445-2454.

Supplementary Information accompanies the paper on Modern Pathology website (http://www.nature.com/ modpathol) 